finger the tumour was found to be fixed to the spine; it was quite smooth and globular in outline. There was no deformity of the nose or displacement of the hard palate or of the anterior or upper wall of the antrum. There were no enlarged glands in the neck. On May 27th the patient was placed under chloroform. Laryngotomy was first performed and a sponge placed in the pbarynx. The soft palate was then divided in the middle line. The tumour was found to fill the naso-pbarynx, to be attached behind to the vertebra, and to extend up into the sphenoidal sinuses. The wall of the pharynx was incised below the tumour and the incision was carried up on each side; with the raspatory and the finger the tumour was then separated from the spine and the skull. The hæmorrhage was slight except at one moment when there was a free flow of venous blood which was easily stopped by pressure. After removal of the tamour the sphenoidal sinuses, basilar process, and anterior arch of the atlas were found to be bare and uneven. The soft palate was united by silkworm gut sutures. A sponge was left in the pharynx over the larynx and the laryngotomy tube was left in. The patient left the operating theatre with a good full pulse. About two hours after the operation there was a rather severe hrmorrbage after a fit of coughing, and the patient vomited about a pint of blood. during which the sponge was ejected from the pharynx. Mr. Irving stopped the bleeding with some difficulty by pressure behind the palate and by the application of tinctura ferri perchloridi. During this the patient became very collapsed, but rallied again. He had nothing by the mouth, but was fed per rectum, his thirst being relieved by an enema of warm water. The nose and mouth were sprayed erery hour night and day - the nose with mercury lotion, and the mouth with boric acid solution. The pulse was 120 and the temperature $962^{\circ} \mathrm{F}$. On the 30 th there had been no more $r æ$ morrhage, but there was slight foetor from the mouth. The patient was allowed a little milk by the mouth, which he took without pain. Since the operation he could hear $v \in \mathrm{ry}$ much better and could breathe well through the nose. He was not allowed to talk. The temperature was $99^{\circ}$, the pulse 92 , and the respirations 14 . On June 1st the patient had no headache or earache, the soft palate had united, and the tongue was clean and moist. He took liquids without any pain and there was no fœtor. On the 3rd the stitches were removed from the palate, and on the next day he was allowed fish diet. The last note, on June 14th, was "The patient has made good progress, takes ordinary diet well, has no discharge and no pain, even when naso-pharynx is irrigated. There is nothing to be felt behind the soft palate. Gjes home to-morrow."

The following report on the tumour has been furnished by Mr. J. Murray, who was surgical registrar at the time:- "On microscopic examination the tumour is found to consist of a groundwork of loose connective tissue containing spindle cells and small round cells, in places undergoing mucoid degeneration. In the section are seen a few ducts cut across ; these are lined with columnar epithelium and contain in their interior round cells held together by mucus. Around these ducts there is marked small-cell infiltration."

\section{CHILDREN'S HOSPITAL, BRIGHTON.}

A CASE OF DILATED URACHUS ACCIDENTALLY OPENED WHILST PEREORMING ABDOMINAI SECTION FOR PERITONITIS; RECOVERY.

(Under the care of Mr. GeobGe MoRGaN.)

CASES of abnormal urachus are rare, but the condition has been described as an occasional complication of median abdominal section. They are not of necessity always centrally placed and their resemblance to ovarian cysts has led to a mistake in diagnosis of some moment. It is stated that hæmorrhages may take place into the cyst in consequence of injury, and symptoms resembling those produced by a twisted ovarian pedicle develop. When there is no history of a discharge from the umbilicus at any time the abnormality is recognised with even more difficulty. In one case recorded by Dr. Frëer, that of a young man aged twenty-four, a cyst was repeatedly tapped, as much as three pints of reddish fluid having been removed on one occasion. At the necropsy a large sac was shown which was evidently a dilated urachus.
A boy aged five years was brought to the hospital on Feb. 18th, 1896, with a two days' history of vomiting anc diarrhoea. The boy was then suffering severe abdomina pain, there was no marked tenderness, his temperature was $102^{\circ} \mathrm{F}$, and his tongue was furred; he had no rash or sore throat. The next day he was much worse; he lay on his left side with his thighs fully flexed. The abdominal wall moved slightly with respiration. The distension, tenderness, and pain were more severe; there was no localised swelling. Sickness and diarrhœea, were almost constant, his temperature was $1036^{\circ}$, and the pulse 108 . He was given small quantities of beef-juice and peptonised milk, and one-twelfth of a grain of morphia hypodermically every four hours. Enemas were not retained. Operation was suggested, but permission could not be obtained. For the next five days there was some improvement in his general condition, pain, and temperature. The abdomen was still distended, but the sickness and diarrhoea were relieved. On the 24th (the seventh day of bis residence in the hospital and the ninth day of his illness) the note was: "Temperature $101^{\circ}$; the pain, tenderness, and distension are still present; the sickness and diarrhoea are better. In the region of the bladder and extending nearly to the umbilicus is a feeling of resistance that is pretty sharply defined; micturition is frequent, but there is no dribbling." Suspecting the swelling to be bladder, a catheter was passed, but only about half an ounce of urine was drawn off ; this did not affect the size or position of the hypogastric fulness. On the 27 th (three days after the above note) the boy's general condition was better, except that he was passing a large quantity of mucus by the bowel (not blood-stained); the distension and hypogastric fulness were less marked. On the evening of the next day-i.e., twelve days from the first symptom-the boy was much worse, the sickness bad returned and the distension was very severe. His temperature was $103^{\circ}$, and his condition looking so critical it was decided to operate at once. At 930 P.M. Dr. Chaffey administered the anæsthetic, and Mr. Morgan, assisted by Dr. Beattie, the house surgeon, opened the abdomen in the middle line, the incision extending from the umbilicus to a point near the pubes. The deeper layers were divided carefully with the director. Whilst opening what was taken for the subperitoneal fat and peritoneum a gush of about one ounce of clear urine escaped. The wound was at once clamped and a catheter was passed. The bladder was found to be quite empty and lying in the pelvis, but the catheter could be passed up to the wound in the cyst, where the clamp was. After carefully dissecting around the cyst the abdominal cavity was opened. There were signs of recent peritonitis, with flakes of lymph, but there was no pus. The abdominal cavity was flushed with hot water, and the intestines were carefully sponged. The boy was too ill to make a prolonged examination or to dissect out the cyst, but it was certain that the cyst was in the middle line, running up to the umbilicus and communicating with the bladder. After washing out the bladder and cyst with boric solution the wound in the latter was closed with a double row of silk sutures, the stitches not penetrating to the mucous membrane. The abdominal wall was also carefully closed. The next day the boy was much better, the sickness had ceased, and the distension was relieved. For the first three days after the operation the wound in the abdominal wall appeared to be healing by first intention, but on the fourth day pus began to well up from the suture wounds. Three stitches were taken out and the pus washed away. For ten days after this some pus and urine escaped by the abdominal wound, but this became less each day and the boy's general condition improved daily. Twenty-six days after the operation the wound was quite closed and the boy was quite well.

Remarks by Mr. MoRgan.-So little reference is made to dilated urachus in our surgical text-books that I presume the condition is a rare one, or rarely discovered except post mortem. I could not see that the dilated urachus bad played any important part in the boy's illness. The peritonitis was evidently secondary to a severe enteritis the cause of which could not be discovered. It is possible that as the distension of the intestines became more marked it prevented the urine in the cyst from escaping into the bladder, for it is certain that when the bladder was emptied by the catheter the hypogastric swelling was unaltered. I should say that the calibre of the urachus before it was opened and the urine drained away was as nearly as possible that of a piece of ileum of a child aged five years. 